\section{Qualidade da dieta e fatores associados entre idosos: estudo de base populacional em Campinas, São Paulo, Brasil}

\author{
Diet quality and associated factors among the \\ elderly: a population-based study in Campinas, \\ São Paulo State, Brazil
}

\author{
Calidad de la dieta y sus factores asociados \\ en ancianos: estudio de base poblacional en \\ Campinas, São Paulo, Brasil
}

Daniela de Assumpção 1

Semíramis Martins Álvares Domene 2

Regina Mara Fisberg 3

Marilisa Berti de Azevedo Barros 1

\footnotetext{
${ }^{1}$ Faculdade de Ciências Médicas, Universidade Estadual de Campinas, Campinas, Brasil.

2 Departamento de Saúde Clínica e Instituições, Universidade Federal de São Paulo, Santos, Brasil. 3 Faculdade de Saúde Pública, Universidade de São Paulo, São Paulo, Brasil.

Correspondência D. Assumpção Departamento de Saúde Coletiva, Faculdade de Ciências Médicas, Universidade Estadual de Campinas.

Rua Tessália Vieira de Camargo 126, Cidade Universitária Zeferino Vaz, Campinas, SP 13083-887, Brasil.

danipos@fcm.unicamp.br
}

\begin{abstract}
The aim of this study was to assess the quality of diet among the elderly and associations with socio-demographic variables, health-related behaviors, and diseases. A population-based cross-sectional study was conducted in a representative sample of 1,509 elderly participants in a health survey in Campinas, São Paulo State, Brazil. Food quality was assessed using the Revised Diet Quality Index (DQI-R). Mean index scores were estimated and a multiple regression model was employed for the adjusted analyses. The highest diet quality scores were associated with age 80 years or older, Evangelical religion, diabetes mellitus, and physical activity, while the lowest scores were associated with home environments shared with three or more people, smoking, and consumption of soft drinks and alcoholic beverages. The findings emphasize a general need for diet quality improvements in the elderly, specifically in subgroups with unhealthy behaviors, who should be targeted with comprehensive strategies.
\end{abstract}

Food Consumption; Diet; Elderly Nutrition; Health of the Elderly

\section{Resumo}

O objetivo foi avaliar a qualidade da dieta de idosos segundo variáveis sociodemográficas, comportamentos relativos à saúde e morbidades. Trata-se de estudo transversal de base populacional em amostra de 1.509 idosos, obtida por inquérito de saúde conduzido em 2008-2009, em Campinas, São Paulo, Brasil. A qualidade alimentar foi avaliada pelo Índice de Qualidade da Dieta Revisado $(I Q D-R)$. Foram estimadas médias do IQD-R segundo as variáveis independentes e desenvolvido modelo de regressão linear múltipla para as análises ajustadas. Os segmentos de idosos que apresentaram escores superiores de qualidade da dieta foram os de 80 anos e mais, evangélicos, que praticavam atividade física de lazer e os diabéticos; escores inferiores foram observados nos que residiam com três ou mais pessoas, nos tabagistas e nos que relataram preferência por refrigerantes e bebidas alcoólicas. Os achados apontam para a necessidade de melhora da qualidade da dieta dos idosos em geral, mas especialmente nos segmentos com outros comportamentos não saudáveis, indicando, ainda, a relevância de intervenções integradas e não focadas em um fator.

Consumo de Alimentos; Dieta; Nutrição do Idoso; Saúde do Idoso 


\section{Introdução}

A população idosa brasileira apresenta elevadas prevalências de excesso de peso $(58,4 \%)$ e obesidade $(19,4 \%) 1$. O processo fisiológico do envelhecimento provoca alterações na composição corporal decorrentes do aumento da massa gorda em detrimento da massa magra, modificações estas, relacionadas ao declínio da atividade física e queda na taxa de metabolismo basal. Mas, além do sobrepeso, parcela significativa dos idosos é acometida por carências nutricionais e mesmo por desnutrição. No idoso, ocorrem alterações na ingestão alimentar provocadas por perda de apetite, diminuição da capacidade gustativa e olfativa, distúrbios de deglutição, o que conduz a menor absorção de vitaminas, minerais e outros nutrientes 2 . Entre os fatores que podem resultar em menor ingestão alimentar, destacam-se ainda o isolamento social, a depressão, a viuvez e as incapacidades 3 . Em decorrência do reduzido consumo de alimentos ou da adoção de uma dieta monótona, o idoso pode apresentar depleção de nutrientes essenciais para a manutenção da saúde e controle das doenças ${ }^{2}$.

Dados recentemente publicados, referentes ao Inquérito Nacional de Alimentação 2008-2009, evidenciam inadequação na dieta dos idosos brasileiros. Foram analisados 4.322 indivíduos com 60 anos ou mais, sendo observadas prevalências elevadas de ingestão inadequada para as vitaminas $\mathrm{A}, \mathrm{C}, \mathrm{D}, \mathrm{E}$, tiamina e piridoxina e para os minerais cálcio, magnésio, zinco e cobre. O consumo habitual de sódio foi excessivo para $80 \%$ dos homens e $61 \%$ das mulheres 4 .

Em face do acelerado envelhecimento populacional e consequente crescimento da carga de doenças crônicas, incapacidades e demanda por serviços de saúde, bem como dos efeitos da inadequada alimentação dos idosos no estado geral de saúde e na incidência de comorbidades, identificar os padrões dietéticos e monitorar a magnitude das mudanças tornam-se tarefas indispensáveis. O Healthy Eating Index (HEI) 5 é um instrumento que viabiliza essas aplicações e permite avaliar a qualidade da dieta em consonância com os avanços científicos no campo da nutrição, que vão sendo incorporados nos guias dietéticos. Com a publicação do Dietary Guidelines for Americans (2005) 6, o HEI foi revisado, passando a ter 12 componentes, que avaliam o grau de atendimento às recomendações de consumo de alimentos e nutrientes 7. No Brasil, o HEI foi adaptado por Fisberg et al. ${ }^{8}$, originando o Índice de Qualidade da Dieta (IQD). Considerando o lançamento das primeiras recomendações oficiais sobre alimentação, dispostas no Guia Alimentar para a População Brasileira 9, o IQD foi atualizado e designado de Índice de Qualidade da Dieta Revisado (IQD-R) 10.

Levando em conta o cenário de crescimento da população idosa e a relevância de diagnosticar e monitorar os padrões de comportamento alimentar deste segmento etário, o presente estudo teve por objetivo avaliar a qualidade global da dieta de idosos de 60 anos e mais, residentes no Município de Campinas, São Paulo, Brasil, segundo variáveis sociodemográficas, de comportamentos de saúde e morbidades.

\section{Métodos}

Trata-se de estudo transversal de base populacional, que utilizou dados provenientes do Inquérito de Saúde no Município de Campinas (ISA-Camp 2008), realizado pelo Centro Colaborador em Análise de Situação de Saúde do Departamento de Saúde Coletiva, Faculdade de Ciências Médicas, Universidade Estadual de Campinas (FCM/ Unicamp). O período de coleta de dados ocorreu entre os meses de fevereiro de 2008 a abril de 2009.

A amostra do inquérito foi selecionada por procedimentos de amostragem probabilística, por conglomerado e em dois estágios: setor censitário e domicílio. No primeiro estágio, foram sorteados cinquenta setores censitários com probabilidade proporcional ao tamanho (número de domicílios). Foram utilizados os setores do Instituto Brasileiro de Geografia e Estatística (IBGE. http://www.ibge.gov.br), definidos para o Censo Demográfico de 2000; considerando o tempo decorrido, foi feito o arrolamento dos domicílios dos setores selecionados para a obtenção de uma listagem atualizada de endereços. No segundo estágio, procedeu-se o sorteio dos domicílios.

O tamanho da amostra foi calculado valendo-se da estimativa de uma prevalência de $50 \%$ (que corresponde à variabilidade máxima), com nível de $95 \%$ de confiança, erro de amostragem entre 4 e 5 pontos percentuais e um efeito de delineamento de 2 , totalizando mil pessoas no estrato de 60 anos e mais. Esperando 80\% de taxa de resposta, o tamanho da amostra foi ampliado para 1.250. Para obter o tamanho desejado, foi definido o número de domicílios que deveriam ser sorteados; com base na razão população idosa/domicilio, foram sorteados 3.900 domicílios para entrevistas com idosos.

As entrevistas foram feitas diretamente com os moradores de 60 anos e mais, de ambos os sexos, não institucionalizados, residentes na área urbana do Município de Campinas.

As informações foram coletadas por meio de um questionário estruturado em 14 blocos te- 
máticos, testado em estudo-piloto e aplicado por entrevistadores treinados e supervisionados. O bloco temático sobre consumo alimentar incluiu o recordatório de 24 horas (R24h), método que consiste no levantamento e quantificação de todos os alimentos e bebidas ingeridos nas 24 horas precedentes à entrevista. O R24h foi aplicado por entrevistadores treinados a orientar o relato dos participantes, com o cuidado de não interrompê-los, e a evitar induzir respostas. As entrevistas foram conduzidas nos diferentes dias da semana e meses do ano para captar a variabilidade interindividual do padrão de consumo alimentar 11 .

\section{Variáveis do estudo}

A variável dependente foi derivada do IQD-R, adaptado do HEI-2005 por Previdelli et al. 10. O IQD-R é constituído por 12 componentes: nove relativos a grupos de alimentos (frutas totais; frutas integrais; vegetais totais; vegetais verdes-escuros e alaranjados e leguminosas; cereais totais; cereais integrais; leite e derivados; carnes, ovos e leguminosas; óleos), dois referentes a nutrientes (sódio e gordura saturada) e um que avalia o percentual energético proveniente das gorduras, saturada e trans, álcool e açúcar de adição (Gord_AA).

Os componentes recebem pontuações específicas que variam de 0 (mínima) a 5, a 10 ou a 20 (máximas), dependendo do componente (Tabela 1). A pontuação mínima é atribuída ao consumo nulo (componentes 1 a 9) ou ao consumo acima do limite preconizado (componentes 10 a 12), enquanto a pontuação máxima de cada componente é estabelecida atingindo ou ultrapassando o valor recomendado de ingestão. Escores para os valores intermediários de ingestão, compreendidos no intervalo entre os critérios de pontuação mínima e máxima, são atribuídos de forma proporcional. Os componentes de 1 a 9 avaliam a adequação e os componentes de 10 a 12, a moderação, o que confere sentidos opostos ao significado das pontuações, ou seja, o aumento do consumo reflete positivamente na pontuação dos grupos 1 a 9 e negativamente nos grupos de 10 a 12. O IQD-R total é representado pela soma dos componentes, podendo atingir o máximo de 100 pontos.

O cálculo do IQD-R baseou-se nas informações obtidas pela aplicação de um R24h. Durante o trabalho de campo, o conteúdo dos recordatórios era verificado minuciosamente pela autora principal deste estudo para identificar e solucionar falhas no preenchimento. Foi realizada a quantificação dos R24h com o propósito de transformar em gramas ou mililitros as quanti- dades de alimentos e preparações referidas em medidas caseiras. Para isso, foram utilizadas informações disponíveis em tabelas de medidas caseiras 12,13, rótulos de alimentos e serviços de atendimento ao consumidor. O valor nutritivo dos alimentos consumidos foi calculado por meio do software Nutrition Data System for Research, versão 2007 (NCC Food and Nutrient Database, University of Minnesota, Estados Unidos). Foi efetuada a análise de consistência dos dados por meio da checagem dos R24h que totalizavam energia inferior a $800 \mathrm{kcal}$ e superior a $3.500 \mathrm{kcal}$.

As variáveis independentes analisadas neste estudo foram:

- Demográficas e socioeconômicas: sexo, idade, raça/cor da pele autorreferida, religião, estado conjugal, número de moradores no domicílio, escolaridade (em anos de estudo), renda familiar mensal per capita (em salários mínimos) e atividade ocupacional.

- Comportamentos relacionados à saúde: atividade física em contexto de lazer avaliada pelo Questionário Internacional de Atividade Física (IPAQ), versão longa ${ }^{14}$, categorizada em - ativos (idosos que praticam ao menos 150 minutos por semana, distribuídos, no mínimo, por três dias), insuficientemente ativos (os que praticam menos de 150 minutos por semana ou mais, porém em menos de três dias na semana) e sedentários (os que não praticam qualquer tipo de atividade física de lazer em nenhum dia da semana) 15; tabagismo e disposição de regras no domicílio sobre o tabagismo; frequência de consumo de bebida alcoólica e relato da bebida de preferência.

- Morbidades: presença de hipertensão, diabetes, número de doenças crônicas entre as incluídas em checklist, transtorno mental comum (TMC) avaliado pelo Self-reporting Questionnarie (SRQ-20), considerando apresentar o problema os indivíduos com escore $\geq 5$ pontos para homens e mulheres 16, autoavaliação da saúde e número de medicamentos utilizados nos três dias anteriores à pesquisa; índice de massa corporal (IMC) calculado por meio de informações autorreferidas de peso e altura. Foram usados os pontos de corte recomendados para idosos 17 com as seguintes classificações: baixo peso $\left(<22 \mathrm{~kg} / \mathrm{m}^{2}\right)$, eutrofia $\left(22-27 \mathrm{~kg} / \mathrm{m}^{2}\right)$ e excesso de peso $\left(>27 \mathrm{~kg} / \mathrm{m}^{2}\right)$.

Para as análises deste estudo, foram estimadas as médias e intervalos de $95 \%$ de confiança (IC95\%) do IQD-R total e de cada componente, bem como o percentual das médias dos componentes em relação ao escore máximo. Os valores médios do IQD-R total segundo as categorias das variáveis independentes foram determinados pelo uso de regressão linear simples, com nível 
Critérios de pontuação dos componentes do Índice de Qualidade da Dieta Revisado (IQD-R).

\begin{tabular}{|c|c|c|c|}
\hline Componentes do IQD-R & Pontuação mínima & Pontuação máxima & Critérios de pontuação \\
\hline \multirow[t]{2}{*}{ 1. Frutas totais * } & 0 & 5 & 0: não consumo \\
\hline & & & 5: 1,0 porção/1.000kcal \\
\hline \multirow[t]{2}{*}{ 2. Frutas integrais } & 0 & 5 & 0: não consumo \\
\hline & & & 5: 0,5 porção/1.000kcal \\
\hline \multirow[t]{2}{*}{ 3. Vegetais totais } & 0 & 5 & 0: não consumo \\
\hline & & & 5: 1,0 porção/1.000kcal \\
\hline \multirow{2}{*}{$\begin{array}{l}\text { 4. Vegetais verdes escuros e } \\
\text { alaranjados e leguminosas }\end{array}$} & 0 & 5 & 0: não consumo \\
\hline & & & 5: 0,5 porção/1.000kcal \\
\hline \multirow[t]{2}{*}{ 5. Cereais totais } & 0 & 5 & 0: não consumo \\
\hline & & & 5: 2,0 porções $/ 1.000 \mathrm{kcal}$ \\
\hline \multirow[t]{2}{*}{ 6. Cereais integrais } & 0 & 5 & 0: não consumo \\
\hline & & & 5: 1,0 porção/1.000kcal \\
\hline \multirow[t]{2}{*}{ 7. Leite e derivados } & 0 & 10 & 0: não consumo \\
\hline & & & 10: 1,5 porção/1.000kcal \\
\hline \multirow[t]{2}{*}{ 8. Carnes, ovos e leguminosas } & 0 & 10 & 0: não consumo \\
\hline & & & 10: 1,0 porção/1.000kcal \\
\hline \multirow[t]{2}{*}{ 9. Óleos ** } & 0 & 10 & 0: não consumo \\
\hline & & & 10: 0,5 porção/1.000kcal \\
\hline \multirow[t]{2}{*}{ 10. Gordura saturada } & 0 & 10 & $0: \geq 15 \%$ do valor energético total \\
\hline & & & $10: \leq 7 \%$ do valor energético total \\
\hline \multirow[t]{2}{*}{ 11. Sódio } & 0 & 10 & $0: \geq 2,0 \mathrm{~g} / 1.000 \mathrm{kcal}$ \\
\hline & & & $10: \leq 0,75 \mathrm{~g} / 1.000 \mathrm{kcal}$ \\
\hline \multirow[t]{2}{*}{ 12. Gord_AA } & 0 & 20 & $0: \geq 35 \%$ do valor energético total \\
\hline & & & $20: \leq 10 \%$ do valor energético total \\
\hline IQD-R total & 0 & 100 & \\
\hline
\end{tabular}

Gord_AA: percentual energético proveniente das gorduras, saturada e trans, álcool e açúcar de adição.

* Representam o consumo de frutas na forma de suco natural;

** Incluem as gorduras das oleaginosas e dos peixes.

Fonte: Previdelli et al. 10.

de significância de 5\%. Foi desenvolvido modelo de regressão linear múltipla em duas etapas. $\mathrm{Na}$ primeira, foram inseridas as variáveis demográficas e socioeconômicas com alguma categoria apresentando nível de significância inferior a 0,20 na análise bivariada, tendo permanecido no modelo aquelas com $\mathrm{p}<0,05$. Na segunda etapa, foram acrescentadas ao modelo as variáveis de comportamentos relacionados à saúde e morbidades que tiveram alguma categoria com $\mathrm{p}<$ 0,20 na análise bivariada, mantendo-se as que permaneceram com nível de 5\% de significância. Todas as etapas do modelo foram ajustadas pela energia total da dieta 18 .

As entrevistas foram digitadas em banco de dados elaborado com o uso do Epidata 3.1 (Epidata Association, Odense, Dinamarca), e as análises estatísticas foram feitas no módulo svy do programa Stata 11.0 (Stata Corp., College Station,
Estados Unidos), que permite a análise de dados de amostras complexas.

O projeto deste estudo foi aprovado pelo Comitê de Ética em Pesquisa da FCM/Unicamp, em adendo ao parecer no 079/2007.

\section{Resultados}

Entre os domicílios sorteados para a amostra de idosos, houve $6,5 \%$ de perda por dificuldade de encontrar um residente na moradia. Dos 1.558 idosos identificados para a realização da pesquisa, $2,5 \%$ recusaram-se a participar, obtendo-se, ao final, um total de 1.519 entrevistas. Destas, dez foram excluídas devido ao não preenchimento do R24h. Portanto, foram analisadas as informações de 1.509 idosos, com idade média de 69,9 anos (IC95\%: 69,3-70,6). 
A definição do tamanho da amostra não se baseou em testes de associação, mas o total de 1.509 idosos seria suficiente para detectar diferenças de, ao menos, 1,6 entre as médias dos escores do IQD-R, considerando um erro tipo I de $5 \%$, um erro beta de $20 \%$, deff de 2 e um tamanho amostral de pelo menos 110 pessoas nas categorias analisadas.

A população estudada é composta por maior proporção de mulheres (57\%), de pessoas com idades entre 60 e 69 anos (53,8\%), de cor branca $(78,1 \%)$ e de religião católica $(66,6 \%) ; 39,9 \%$ tinham entre quatro e oito anos de escolaridade; $39,1 \%$ tinham menos de um salário mínimo de renda familiar mensal per capita e 51,3\% eram aposentados. Idosos sedentários em contexto de lazer representaram $67,7 \%$; $11,5 \%$ eram fumantes; $9,9 \%$ ingeriam álcool numa frequência maior ou igual a duas vezes por semana e $21,7 \%$ eram diabéticos.

A média estimada de pontos do IQD-R foi de 62,4 (IC95\%: 61,7-63,1), sendo 61,8 (IC95\%: 60,063,6) para os homens e 62,2 (IC95\%: 61,5-63,7) para as mulheres. Os componentes do IQD-R com piores pontuações foram os cereais integrais, sódio, leite e derivados, frutas totais e frutas integrais (Tabela 2).

Os escores médios do IQD-R foram superiores nos indivíduos com 80 anos e mais, nos adeptos da religião evangélica, no segmento de renda per capita igual ou superior a quatro salários mí- nimos e nas donas de casa. Por outro lado, escore inferior foi verificado em idosos que residiam em domicílios com três ou mais moradores, comparados aos que relataram morar sozinhos ou com apenas um outro morador (Tabela 3).

Os indivíduos classificados como ativos ou insuficientemente ativos no lazer tiveram pontuações médias do IQD-R significativamente maiores quando comparados aos sedentários. Escores significativamente inferiores foram detectados entre os fumantes e os que viviam em residências onde o tabagismo era permitido livremente. Entre os que ingeriam bebidas alcoólicas, foi observado gradiente de piora dos escores com o aumento da frequência de consumo. Os idosos que relataram preferência por refrigerantes e por bebidas alcoólicas apresentaram médias menores em relação aos que relataram água ou chá como a bebida de preferência (Tabela 4).

Dietas de melhor qualidade foram constatadas entre os idosos que referiram o diagnóstico de diabetes, a presença de três ou mais doenças crônicas e o uso de três ou mais medicamentos nos três dias prévios à entrevista (Tabela 5).

Os resultados da análise de regressão linear múltipla (Tabela 6) mostram médias de pontos significativamente superiores entre os indivíduos de 80 anos e mais, evangélicos, que praticavam algum nível de atividade física no lazer e aqueles com diabetes. Idosos fumantes, os que compartilhavam a residência com três ou mais

Tabela 2

Médias da pontuação dos componentes do Índice de Qualidade da Dieta Revisado (IQD-R) e percentual de atendimento ao escore máximo na população de 60 anos e mais. Inquérito de Saúde de Campinas (ISA-Camp 2008).

\begin{tabular}{lccc}
\hline Componentes do IQD-R & $\begin{array}{c}\text { Pontuação máxima } \\
\text { Frutas totais }\end{array}$ & $\begin{array}{c}\text { Média da pontuação } \\
\text { (IC95\%) }\end{array}$ & $\begin{array}{c}\text { Percentual em relação } \\
\text { ao escore máximo }\end{array}$ \\
\hline Frutas integrais & 5 & $2,6(2,4-2,8)$ & 52,0 \\
Vegetais totais & 5 & $2,9(2,7-3,0)$ & 58,0 \\
Vegetais verdes escuros e alaranjados e & 5 & $4,5(4,4-4,6)$ & 90,0 \\
leguminosas & 5 & $3,9(3,8-4,0)$ & 78,0 \\
Cereais totais & & & 94,0 \\
Cereais integrais & 5 & $4,7(4,6-4,7)$ & 8,0 \\
Leite e derivados & 5 & $0,4(0,3-0,4)$ & 49,0 \\
Carnes, ovos e leguminosas & 10 & $4,9(4,5-5,2)$ & 87,0 \\
Óleos & 10 & $8,7(8,5-8,8)$ & 87,0 \\
Gordura saturada & 10 & $8,7(8,5-8,9)$ & 65,0 \\
Sódio & 10 & $6,5(6,3-6,8)$ & 21,0 \\
Gord_AA & 10 & $2,1(1,9-2,2)$ & 62,5 \\
IOD-R total & 20 & $12,5(12,0-12,9)$ & 100,0 \\
\hline Gord_AA prio & $62,4(61,7-63,1)$ & \\
\hline
\end{tabular}

Gord_AA: percentual energético proveniente das gorduras, saturada e trans, álcool e açúcar de adição. 
Tabela 3

Médias do Índice de Qualidade da Dieta Revisado (IQD-R), segundo variáveis sociodemográficas na população de 60 anos e mais. Inquérito de Saúde de Campinas (ISA-Camp 2008).

\begin{tabular}{|c|c|c|c|c|}
\hline Variáveis & $\mathrm{n}$ & $\%$ & Médias brutas (IC95\%) & Valor de $p$ \\
\hline \multicolumn{5}{|l|}{ Sexo } \\
\hline Masculino * & 611 & 43,0 & $61,8(60,0-63,6)$ & \\
\hline Feminino & 898 & 57,0 & $62,2(61,5-63,7)$ & 0,476 \\
\hline \multicolumn{5}{|l|}{ Faixa etária (anos) } \\
\hline $60-69 *$ & 812 & 53,8 & $61,8(60,9-62,7)$ & \\
\hline $70-79$ & 498 & 32,9 & $62,7(60,4-64,9)$ & 0,222 \\
\hline $80 e+$ & 199 & 13,3 & $64,3(61,3-67,3)$ & $0,020 * \star$ \\
\hline \multicolumn{5}{|l|}{ Raça/Cor da pele ${ }^{\star \star \star}$} \\
\hline Branca * & 1.147 & 78,1 & $62,4(61,6-63,2)$ & \\
\hline Preta & 108 & 7,2 & $60,7(56,9-61,1)$ & 0,104 \\
\hline Parda & 223 & 14,7 & $63,2(62,4-65,7)$ & 0,315 \\
\hline \multicolumn{5}{|l|}{ Religião } \\
\hline Católica * & 1.001 & 66,6 & $61,8(60,9-62,7)$ & \\
\hline Evangélica & 333 & 21,6 & $63,4(61,3-65,5)$ & $0,011 \star \star$ \\
\hline Outras & 83 & 5,7 & $64,1(59,5-68,8)$ & 0,230 \\
\hline Sem religião & 89 & 6,1 & $63,4(60,2-66,7)$ & 0,178 \\
\hline \multicolumn{5}{|l|}{ Estado conjugal } \\
\hline Com cônjuge * & 837 & 56,4 & $62,3(61,4-63,2)$ & \\
\hline Separado & 120 & 7,9 & $62,0(59,4-64,0)$ & 0,800 \\
\hline Viúvo & 452 & 29,1 & $63,0(62,3-65,0)$ & 0,323 \\
\hline Solteiro & 100 & 6,6 & $61,7(58,3-63,9)$ & 0,656 \\
\hline \multicolumn{5}{|l|}{ Número de pessoas no domicílio } \\
\hline $1-2$ * & 820 & 54,6 & $63,3(62,3-64,3)$ & \\
\hline $3 e+$ & 689 & 45,4 & $61,4(57,9-61,0)$ & $0,015 * \star$ \\
\hline \multicolumn{5}{|l|}{ Escolaridade (anos) } \\
\hline $0-3$ * & 545 & 35,0 & $63,0(61,7-64,3)$ & \\
\hline $4-8$ & 608 & 39,9 & $61,9(59,1-62,3)$ & 0,157 \\
\hline $9 e+$ & 355 & 25,1 & $62,5(60,0-63,9)$ & 0,589 \\
\hline \multicolumn{5}{|l|}{ Renda per capita (salários mínimos) } \\
\hline$<1 *$ & 602 & 39,1 & $61,8(60,8-62,8)$ & \\
\hline$\geq 1 \mathrm{a}<2,5$ & 569 & 37,3 & $62,8(60,5-65,1)$ & 0,143 \\
\hline$\geq 2,5 a<4$ & 163 & 11,1 & $61,8(58,4-65,2)$ & 0,996 \\
\hline$\geq 4$ & 175 & 12,5 & $63,7(60,8-66,5)$ & 0,054 ** \\
\hline \multicolumn{5}{|l|}{ Atividade ocupacional } \\
\hline Em atividade (inclusive aposentados) * & 313 & 21,8 & $61,4(60,1-62,7)$ & \\
\hline Aposentado & 749 & 51,3 & $62,6(62,2-65,4)$ & 0,145 \\
\hline Dona de casa & 414 & 26,9 & $63,2(63,2-66,7)$ & 0,048 ** \\
\hline
\end{tabular}

* Categoria de referência utilizada para comparação;

** Valor de $\mathrm{p}<0,05$;

*** Excluídos os idosos que se declararam amarelos $(n=25)$ e indígenas $(n=4)$.

pessoas e que tinham os refrigerantes e as bebidas alcoólicas como preferências apresentaram escores médios significativamente menores.

\section{Discussão}

Os principais achados deste estudo apontam para melhor padrão de qualidade da dieta em determinados segmentos da população, como nos idosos com 80 anos ou mais, evangélicos, 
Médias do Índice de Qualidade da Dieta Revisado (IQD-R), segundo variáveis de comportamentos relacionados à saúde na população de 60 anos e mais. Inquérito de Saúde de Campinas (ISA-Camp 2008).

\begin{tabular}{|c|c|c|c|c|}
\hline Variáveis & $\mathbf{n}$ & $\%$ & $\begin{array}{l}\text { Médias brutas } \\
\text { (IC95\%) }\end{array}$ & Valor de $p$ \\
\hline \multicolumn{5}{|l|}{ Atividade física em contexto de lazer (IPAQ) } \\
\hline Sedentário * & 996 & 65,4 & $61,4(60,5-62,3)$ & \\
\hline Insuficientemente ativo & 188 & 12,5 & $65,2(62,4-67,9)$ & $0,000 * *$ \\
\hline Ativo & 325 & 22,1 & $63,9(61,2-66,6)$ & $0,007 * \star$ \\
\hline \multicolumn{5}{|l|}{ Tabagismo } \\
\hline Nunca fumou * & 1.031 & 67,7 & $62,8(61,9-63,7)$ & \\
\hline Ex-fumante & 305 & 20,8 & $63,0(61,4-65,0)$ & 0,831 \\
\hline Fumante & 172 & 11,5 & $59,2(53,7-57,5)$ & $0,000 * \star$ \\
\hline \multicolumn{5}{|l|}{ Regras do domicílio quanto ao tabagismo } \\
\hline Não é permitido fumar em nenhum lugar * & 607 & 40,2 & $63,3(62,3-64,4)$ & \\
\hline É permitido fumar em alguns lugares ou horários & 298 & 19,8 & $62,4(59,8-63,1)$ & 0,245 \\
\hline $\begin{array}{l}\text { É permitido fumar em qualquer lugar/Não existem } \\
\text { regras sobre isso }\end{array}$ & 600 & 40,0 & $61,5(58,1-61,1)$ & $0,016 * \star$ \\
\hline \multicolumn{5}{|l|}{ Frequência de consumo de álcool } \\
\hline Não bebe * & 1.055 & 69,1 & $63,1(62,4-63,9)$ & \\
\hline $1-4$ vezes no mês & 308 & 21,0 & $61,4(58,1-61,3)$ & $0,032 * \star$ \\
\hline 2 ou + vezes por semana & 141 & 9,9 & $59,5(53,9-57,9)$ & $0,001 * \star$ \\
\hline \multicolumn{5}{|l|}{ Bebida de preferência } \\
\hline Água/Chá * & 453 & 30,8 & $63,4(62,2-64,6)$ & \\
\hline Refrigerantes (todos os tipos) & 338 & 22,6 & $60,6(56,0-59,6)$ & $0,003 * \star$ \\
\hline Leite/logurte/Vitaminas & 51 & 3,4 & $63,6(59,6-68,0)$ & 0,918 \\
\hline Café/Bebidas com café & 99 & 6,5 & $62,7(59,3-64,6)$ & 0,576 \\
\hline Suco natural (inclusive água de coco) & 332 & 22,1 & $64,3(63,8-66,7)$ & 0,206 \\
\hline Refrescos & 44 & 2,9 & $62,4(57,2-65,7)$ & 0,650 \\
\hline Bebidas alcoólicas & 169 & 11,7 & $59,6(53,8-57,8)$ & $0,000 * \star$ \\
\hline
\end{tabular}

IPAQ: Questionário Internacional de Atividade Física.

* Categoria de referência utilizada para comparação;

** Valor de $\mathrm{p}<0,05$.

diabéticos e nos que praticam atividade física em contexto de lazer; por outro lado, os resultados verificam pior qualidade alimentar entre os idosos que residem em domicílios com três ou mais pessoas, tabagistas e os que relatam predileção por refrigerantes e bebidas alcoólicas. Observou-se, também, que os componentes do IQD-R que apresentaram as piores pontuações, indicando um consumo inadequado, foram os relativos a cereais integrais, sódio, leite e derivados e frutas.

Na presente pesquisa, o escore médio do IQD-R foi de 62,4 (IC95\%: 61,7-63,1), sendo um pouco inferior ao valor obtido em pesquisa norte-americana que constatou pontuação de 63,8 no estrato de 60 anos e mais 19. No Município de São Paulo, os idosos avaliados no ano de 2008 apresentaram 62,8 pontos, valor que é bastante próximo ao observado em Campinas 20 . A pon- tuação encontrada é superior a 51, sendo as dietas com escores abaixo desse valor consideradas inadequadas, segundo a classificação proposta por Bowman et al. 21. Contudo, a pontuação obtida encontra-se em patamar bem inferior a 80 pontos, que delimita a situação de dieta saudável de acordo com esse autor. O resultado sinaliza dieta que precisa de modificação e que há muito a avançar na melhoria da qualidade da alimentação dos idosos.

Os resultados desta pesquisa indicam piores pontuações nos componentes de cereais integrais, sódio, leite e derivados, frutas totais e frutas integrais. Ao analisarem idosos com 65 anos ou mais incluídos no National Health and Nutrition Examination Survey (NHANES), Juan et al. 22 e Hiza et al. 23 também encontraram escores baixos para grãos integrais, sódio e leite, no entanto a pontuação de frutas totais foi bastante elevada 
Médias do Índice de Qualidade da Dieta Revisado (IQD-R), segundo morbidades, estado de saúde, índice de massa corporal (IMC) e uso de medicamentos em idosos de 60 anos e mais. Inquérito de Saúde de Campinas (ISA-Camp 2008).

\begin{tabular}{|c|c|c|c|c|}
\hline Variáveis & $\mathbf{n}$ & $\%$ & Médias brutas (IC95\%) & Valor de $\mathrm{p}$ \\
\hline \multicolumn{5}{|l|}{ Hipertensão arterial } \\
\hline Não * & 695 & 46,7 & $61,9(60,9-62,9)$ & \\
\hline Sim & 814 & 53,3 & $62,9(62,8-65,0)$ & 0,066 \\
\hline \multicolumn{5}{|l|}{ Diabetes melitus } \\
\hline Não * & 1.176 & 78,3 & $61,7(60,9-62,4)$ & \\
\hline Sim & 331 & 21,7 & $65,2(67,4-69,9)$ & $0,000 * \star$ \\
\hline \multicolumn{5}{|c|}{ Número de doenças crônicas } \\
\hline 0 * & 284 & 19,4 & $61,6(60,2-62,9)$ & \\
\hline $1-2$ & 703 & 47,5 & $61,6(60,1-63,2)$ & 0,945 \\
\hline $3 e+$ & 500 & 33,1 & $64,0(64,8-67,9)$ & $0,003 * \star$ \\
\hline \multicolumn{5}{|l|}{ Transtorno mental comum } \\
\hline Não * & 1.030 & 69,0 & $62,3(61,4-63,2)$ & \\
\hline Sim & 477 & 31,0 & $62,7(60,4-64,9)$ & 0,592 \\
\hline \multicolumn{5}{|l|}{ Autoavaliação da saúde } \\
\hline Excelente/Muito boa & 357 & 24,3 & $63,2(61,9-64,4)$ & \\
\hline Boa & 966 & 63,6 & $62,0(59,2-62,5)$ & 0,161 \\
\hline Ruim/Muito ruim & 186 & 12,1 & $63,2(61,1-65,3)$ & 0,973 \\
\hline \multicolumn{5}{|l|}{$\mathrm{IMC}\left(\mathrm{kg} / \mathrm{m}^{2}\right)$} \\
\hline$<22$ & 251 & 16,5 & $62,2(60,2-63,3)$ & 0,590 \\
\hline $22-27$ * & 681 & 45,6 & $62,6(61,7-63,4)$ & \\
\hline$>27$ & 577 & 37,9 & $62,5(61,0-63,7)$ & 0,823 \\
\hline \multicolumn{5}{|c|}{ Número de medicamentos utilizados nos últimos três dias } \\
\hline 0 * & 289 & 19,5 & $61,4(60,1-62,6)$ & \\
\hline $1-2$ & 554 & 36,9 & $61,8(60,9-63,6)$ & 0,499 \\
\hline $3-5$ & 480 & 31,6 & $63,0(63,2-66,0)$ & 0,030 ** \\
\hline 6 ou + & 182 & 12,0 & $64,5(65,4-69,8)$ & 0,006 ** \\
\hline
\end{tabular}

* Categoria de referência utilizada para comparação;

** Valor de $\mathrm{p}<0,05$

e a de frutas integrais atingiu o valor máximo. Dados do Vigitel 2010 (Vigilância de Fatores de Risco e Proteção para Doenças Crônicas por Inquérito Telefônico) mostram que a ingestão de frutas e hortaliças tende a aumentar com a idade, mas é muito inferior ao mínimo de $400 \mathrm{~g} / \mathrm{dia}$ recomendado pela Organização Mundial da Saúde (OMS) 1 . O governo brasileiro definiu um conjunto de estratégias para elevar o consumo de frutas e vegetais e diminuir o de sal, destacando-se o crescimento da produção, oferta e queda dos preços de alimentos in natura, além de estabelecer acordos com o setor produtivo para reduzir o teor de sal dos produtos industrializados 24 . Também são necessárias ações de incentivo ao consumo de cereais integrais e leite e derivados com baixo teor de gordura, divulgando informação sobre os benefícios que trazem à saúde e adotando medidas fiscais que resultem no barateamento destes alimentos.
Não foi observado, neste estudo, diferença na qualidade da dieta entre os sexos, confirmando o achado de pesquisa desenvolvida na população norte-americana de 65 anos e mais ${ }^{23}$. Entretanto, estudos que analisaram amostras constituídas por adultos e idosos encontraram pontuações significativamente superiores nas mulheres $19,25,26$. A semelhante qualidade da dieta entre homens e mulheres idosos poderia ser explicada pela situação mais comum nessa etapa da vida, com a de estarem aposentados e fazerem, com maior frequência, as refeições no próprio domicílio. Por outro lado, o aumento de doenças paralelamente ao avanço da idade em ambos os sexos tende a modificar e melhorar a qualidade da alimentação.

Ao analisar os escores do IQD-R por faixa etária, verificou-se média significativamente superior no subgrupo dos muito idosos (80 anos e mais). Hiza et al. ${ }^{23}$ encontraram média mais 
Tabela 6

Modelos de regressão linear múltipla: variáveis associadas às médias globais do Índice de Qualidade da Dieta Revisado (IQD-R) em idosos de 60 anos e mais. Inquérito de Saúde de Campinas (ISA-Camp 2008).

\begin{tabular}{|c|c|c|c|c|}
\hline \multirow[t]{2}{*}{ Variáveis } & \multicolumn{2}{|c|}{ Primeira etapa * } & \multicolumn{2}{|c|}{ Segunda etapa ** } \\
\hline & Média (IC95\%) & Valor de $p$ & Média (IC95\%) & Valor de $p$ \\
\hline Energia (kcal) & $65,1(62,0-68,1)$ & & $65,4(61,8-69,1)$ & \\
\hline \multicolumn{5}{|l|}{ Sexo } \\
\hline Masculino *** & $65,1(62,0-68,1)$ & & $65,4(61,8-69,1)$ & \\
\hline Feminino & $64,8(60,7-68,9)$ & 0,664 & $64,6(59,6-69,5)$ & 0,218 \\
\hline \multicolumn{5}{|l|}{ Faixa etária (anos) } \\
\hline $60-69 * * \star$ & $65,1(62,0-68,1)$ & & $65,4(61,8-69,1)$ & \\
\hline $70-79$ & $65,7(61,4-70,1)$ & 0,311 & $65,6(60,7-70,5)$ & 0,858 \\
\hline 80 e + & $67,3(62,3-72,4)$ & $0,031 \#$ & $67,7(62,1-73,3)$ & $0,028 \#$ \\
\hline \multicolumn{5}{|l|}{ Religião } \\
\hline 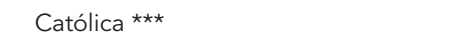 & $65,1(62,0-68,1)$ & & $65,4(61,8-69,1)$ & \\
\hline Evangélica & $66,9(62,7-71,0)$ & $0,002 \#$ & $67,2(62,5-71,9)$ & $0,002 \#$ \\
\hline Outras & $67,2(60,5-73,9)$ & 0,250 & $67,0(60,0-74,1)$ & 0,357 \\
\hline Sem religião & $66,9(61,4-72,3)$ & 0,140 & $66,6(60,4-72,7)$ & 0,364 \\
\hline \multicolumn{5}{|l|}{ Número de pessoas no domicílio } \\
\hline $1-2 * \star \star$ & $65,1(62,0-68,1)$ & & $65,4(61,8-69,1)$ & \\
\hline $3 e+$ & $63,3(58,7-67,8)$ & $0,020 \#$ & $63,8(58,7-68,9)$ & $0,021 \#$ \\
\hline \multicolumn{5}{|l|}{ Atividade física em contexto de lazer (IPAQ) } \\
\hline 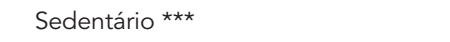 & & & $65,4(61,8-69,1)$ & \\
\hline Insuficientemente ativo & & & $69,1(63,5-74,7)$ & $0,000 \#$ \\
\hline Ativo & & & $68,2(62,8-74,0)$ & $0,002 \#$ \\
\hline \multicolumn{5}{|l|}{ Tabagismo } \\
\hline Nunca fumou $* \star \star$ & & & $65,4(61,8-69,1)$ & \\
\hline Ex-fumante & & & $65,8(60,0-71,5)$ & 0,781 \\
\hline Fumante & & & $63,4(57,9-68,8)$ & $0,022 \#$ \\
\hline \multicolumn{5}{|l|}{ Bebida de preferência } \\
\hline 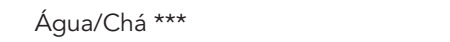 & & & $65,4(61,8-69,1)$ & \\
\hline Refrigerantes (todos os tipos) & & & $62,9(57,4-68,3)$ & $0,005 \#$ \\
\hline Leite/logurte/Vitaminas & & & $65,3(57,6-72,9)$ & 0,946 \\
\hline Café/Bebidas com café & & & $64,9(58,6-71,0)$ & 0,613 \\
\hline Suco natural (inclusive água de coco) & & & $66,3(61,1-71,5)$ & 0,250 \\
\hline Refrescos & & & $64,4(56,7-72,2)$ & 0,625 \\
\hline Bebidas alcoólicas & & & $61,7(56,0-67,4)$ & $0,001 \#$ \\
\hline \multicolumn{5}{|l|}{ Diabetes } \\
\hline 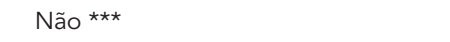 & & & $65,4(61,8-69,1)$ & \\
\hline Sim & & & $69,1(64,3-73,9)$ & $0,000 \#$ \\
\hline
\end{tabular}

IPAQ: Questionário Internacional de Atividade Física.

* Ajustada por energia e pelas variáveis demográficas e socioeconômicas;

** Ajustada por energia e por todas as variáveis da tabela;

*** Categoria de referência utilizada para comparação;

\# Valor de $\mathrm{p}<0,05$. 
elevada no escore total do HEI-2005 e nos componentes fruta total, grãos integrais, leite e energia obtida das gorduras sólidas, álcool e açúcar de adição entre idosos com 75 anos, quando comparados aos de 65 a 74 anos. No estudo de Ervin 19, os indivíduos com 60 anos e mais alcançaram média de pontos superior comparados aos adultos, em virtude do maior consumo dos componentes fruta total, fruta integral, vegetais totais, vegetais verdes escuros e alaranjados e leguminosas, grãos integrais e, também, da menor ingestão de gorduras sólidas, álcool e açúcar de adição. No Brasil, resultados do inquérito Vigitel 2010 mostraram menor exposição a fatores de risco, tais como a ingestão de refrigerantes, de carnes com gordura aparente e do uso abusivo de bebidas alcoólicas; em relação aos fatores de proteção, maior prevalência de consumo recomendado de frutas e hortaliças nas pessoas com 65 anos e mais comparadas às de 18 a 24 anos 1. Em estudo de base populacional, conduzido na Região Metropolitana de Belo Horizonte, Minas Gerais, LimaCosta 27 observou que a ingestão diária de cinco ou mais porções de frutas, verduras e legumes foi $54 \%$ maior nos sujeitos com 60 anos e mais, quando comparados aos de 20 a 29 anos. A melhor qualidade da dieta dos idosos, especialmente do segmento mais velho, pode ser resultante do impacto das doenças crônicas e das comorbidades no estado de saúde, forçando o indivíduo a aderir a um tratamento em que a alimentação saudável é um constituinte fundamental.

Nesta pesquisa, os seguidores da religião evangélica apresentaram pontuação significativamente superior em relação aos católicos. O levantamento de informações referentes ao perfil religioso da população brasileira, obtidas desde 1872 pelos censos demográficos, mostra que, até a década de 1970, mais de $90 \%$ da população seguiam a doutrina católica, mas, atualmente, os católicos somam $64,6 \%$ e os evangélicos, $22,2 \%$ do contingente populacional 28 . Sarri et al. 29 encontraram diferenças importantes entre as práticas alimentares de grupos religiosos (muçulmano, adventista do sétimo dia, cristão ortodoxo, judeu, budista e outras que incluem católicos, testemunha de Jeová e amish). Os autores concluem que a religião é um fator que influencia, além do comportamento alimentar, o relativo ao uso do tabaco, álcool e outras drogas. Não foi encontrado nenhum estudo que tenha analisado a qualidade da dieta nas diferentes categorias religiosas, o que sinaliza a necessidade de investigação deste tema em futuras pesquisas.

Foi encontrado escore significativamente inferior entre os idosos que residem com três ou mais pessoas, comparativamente aos que viviam sozinhos ou com apenas um outro morador. De acordo com o Censo Demográfico de 2010, dos domicílios particulares habitados somente por idosos, 46,6\% eram compostos por uma ou duas pessoas e 53,4\%, por três ou mais 30 . Em Campinas, a distribuição do número de moradores na residência segundo a renda familiar per capita, revelou que as famílias mais numerosas tendem a ter menor renda, o que poderia explicar a pior qualidade alimentar. Não foi encontrado um estudo que tenha relacionado qualidade da dieta com o arranjo familiar, todavia alguns autores detectaram que idosos que moram sozinhos apresentam melhor autoavaliação da saúde 31,32. É possível que estes idosos tenham melhores condições físicas 32, melhor situação financeira, maior autonomia para escolher e preparar os alimentos que desejam, enquanto os indivíduos que moram junto a outros familiares poderiam não ter as mesmas opções.

Em relação aos sedentários, os idosos que praticavam algum nível de atividade física em contexto de lazer tiveram escores mais elevados do IQD-R. Evidências indicam que os idosos fisicamente ativos apresentam menores taxas de mortalidade por todas as causas de óbito, têm níveis mais elevados de saúde funcional, melhor função cognitiva e menor risco de queda 15 . Em pesquisa que avaliou a qualidade global da dieta e o desempenho físico de indivíduos com 60 anos e mais, participantes do NHANES 19992002 33, foi constatada associação entre pontuações globais mais elevadas do HEI-2005 com maior velocidade de marcha e maior força extensora do joelho. No Brasil, resultados do Vigitel 2010 mostram que a prevalência de atividade física no lazer em indivíduos com 65 anos e mais é de 12,2\% (IC95\%: 10,7-13,8), com diferencial significativo entre homens (16,6\%; IC95\%: 13,7-19,6) e mulheres (9,4\%; IC95\%: 7,7-11,1) 1. Aumentar a prevalência de atividade física no lazer é uma das metas nacionais contempladas no Plano de Ações Estratégicas para o Enfrentamento das Doenças Crônicas Não Transmissíveis (2011-2022), e, para isso, o governo se apoia em ações como o Programa Academia da Saúde, Programa Saúde na Escola, na construção e reativação de espaços urbanos destinados à realização de atividade física, entre outras 24 .

Confrontados aos idosos que nunca fumaram, os tabagistas apresentaram pior qualidade da dieta. O tabagismo é amplamente reconhecido como fator de risco para doenças respiratórias, cardiovasculares, neoplasias, tuberculose e outras ${ }^{34}$. O uso e a exposição ao tabaco respondem por aproximadamente seis milhões de óbitos/ano no mundo e estima-se que em 2020 representem $10 \%$ de todas as causas de morte 34 . Em pouco mais de duas décadas, o Brasil con- 
quistou reduções substanciais na prevalência do tabagismo, resultante de medidas regulatórias, educativas e legislativas, adesão ao Tratado Convenção-Quadro para o Controle do Tabaco, entre outras 24,35. A prevalência de fumantes com 18 anos e mais declinou de 35\% em 1989 para 16\% em 2006 36, e um recente estudo publicado com dados do Vigitel 20101 apresenta um valor de $15,1 \%$. Na pesquisa de Xu et al. 33 , comparativamente aos que nunca fumaram, os idosos fumantes tiveram pontuação significativamente inferior do HEI-2005. McClernon et al. 37 investigaram as alterações produzidas por alimentos e bebidas na palatabilidade do cigarro, numa amostra de 209 adultos fumantes. Frutas e vegetais, leite, produtos lácteos e bebidas não cafeinadas (inclusive água) foram relacionados à piora do sabor do cigarro; por outro lado, as carnes e as bebidas cafeinadas e alcoólicas realçaram as qualidades sensoriais do tabaco. Outros pesquisadores 38 avaliaram a relação entre o consumo de frutas e vegetais, medido em quartil, com indicadores de dependência e abstinência de nicotina. Foi verificado que o maior grau de dependência, avaliado por fumar vinte ou mais cigarros por dia, fumar nos primeiros trinta minutos após acordar e obter pontuação $\geq 9$ na $E s$ cala de Síndrome de Dependência de Nicotina, esteve atrelado ao menor consumo de frutas e vegetais. Entre os indicadores de abstinência, a não utilização de produtos contendo tabaco, por ao menos trinta dias, foi três vezes superior naqueles que ingeriam mais frutas e vegetais. Estes achados sinalizam que as estratégias de cessação do tabagismo poderiam agregar recomendações sobre a dieta, estimulando o consumo de frutas, vegetais e leite, bem como a redução do consumo de carne e álcool.

Nossos resultados apontam piores escores de qualidade da dieta entre os idosos que relataram preferência por refrigerantes e bebidas alcoólicas, em relação aos que disseram gostar mais de água ou chá. Refrigerantes integram a categoria de alimentos ultraprocessados, de "natureza intrinsecamente não saudável” por sua elevada concentração de açúcar e adição de conservantes, corantes e flavorizantes 39 . Resultados da Pesquisa de Orçamentos Familiares (POF) 2008-2009 mostram quedas nas prevalências e no consumo médio per capita de refrigerantes com o avançar da idade 40. Entretanto, no estudo de Bueno et al. 41 , foi constatado que, seguido do açúcar de mesa, os refrigerantes representaram a maior fonte de açúcar de adição da dieta de idosos. Thomson et al. 42 simularam os efeitos de $25 \%, 50 \%$ e $100 \%$ de substituição de bebidas açucaradas por água na qualidade da dieta e na ingestão energética total de adultos norte-americanos. $\mathrm{O}$ maior nível de substituição resultaria, por um lado, em acréscimos de 3,8 pontos no escore total do HEI2005 e 4,4 pontos no componente composto por gorduras sólidas, bebidas alcoólicas e açúcar de adição, e em uma redução de $11 \%$ na média de energia diária consumida pela população.

Os idosos que mencionaram preferência por bebidas alcoólicas tiveram 3,8 pontos a menos no IQD-R total em relação aos que referiram água ou chá. A predileção por bebida alcoólica não significa que o indivíduo tenha um consumo excessivo de álcool e, na população em estudo, observou-se que menos de $10 \%$ referiram frequência de ingestão igual ou superior a duas vezes na semana. A associação entre o consumo de bebida alcoólica e a qualidade da alimentação, medida pelo HEI-2005, foi testada por Breslow et al. 43 em adultos norte-americanos participantes do NHANES 1999-2006. Com o aumento do número de doses ingeridas por dia, os autores verificaram gradiente de piora das médias de pontos no escore total do HEI-2005 e, também, nos componentes fruta total, fruta integral, energia das gorduras sólidas, álcool e açúcar de adição, em ambos os sexos. Analisando homens adultos, Herbeth et al. ${ }^{44}$ constataram que o crescente consumo de álcool acarretava menor ingestão de frutas, vegetais, leite, iogurte e queijo fresco, e maior consumo de batatas, carnes e queijo. $\mathrm{O}$ pior escore do IQD-R no segmento que consome mais álcool poderia ser explicado pelo elevado conteúdo energético das bebidas alcoólicas e pelos tipos de alimentos usualmente escolhidos para acompanhar o seu consumo 43.

Os resultados obtidos nesta pesquisa atestam a concomitância de uma dieta de má qualidade com outros comportamentos não saudáveis, como o sedentarismo, tabagismo e a predileção por refrigerantes e bebidas alcoólicas. Dessa forma, ações dirigidas à promoção da saúde dos idosos não devem focar um único fator, como, por exemplo, a alimentação saudável, mas, sim, o conjunto de fatores que impactam no estado geral de saúde, por meio de intervenções mais amplas.

Melhor qualidade da dieta foi verificada nos idosos que relataram presença de diabetes. Estudos demonstram o crescimento acentuado da prevalência de diabetes no Brasil. No período de 2003 a 2008 , aumentou $37 \%$ (de $2,49 \%$ para $3,59 \%$ ) entre os adultos com 18 anos e mais 45; particularmente entre os idosos, cresceu $26 \%$ de 1998 a 2003 e $56 \%$ de 1998 a 2008 46. Chen et al. 26, estudando adultos de 20 a 65 anos, verificaram pontuação significativamente superior do HEI2005 no grupo dos diabéticos, havendo maior consumo de frutas e vegetais e menor ingestão de energia. 
Outros autores observaram gradiente de melhora dos escores do HEI-2005 com o aumento do número de morbidades 25. Segundo Barros et al. 45, o reconhecimento da doença pelo indivíduo depende do acesso aos serviços de saúde e aos testes diagnósticos. O maior acesso aos serviços, especialmente após o diagnóstico, ocasionaria oportunidades mais frequentes de orientações sobre as condutas de controle das doenças, incluindo as referentes à alimentação. Por outro lado, receber um diagnóstico de doença crônica tende a provocar nos pacientes o reconhecimento da necessidade de mudanças no estilo de vida com a adoção de hábitos mais saudáveis.

Uma das limitações do presente estudo decorre da aplicação de apenas um R24h, o que não capta a ingestão usual do indivíduo, em face da variabilidade de consumo entre os dias da semana. No entanto, quando aplicado em base populacional e de forma a considerar os diferentes dias da semana e as estações do ano, como ocorreu no ISA-Camp 2008, pode-se estimar uma média de ingestão para a populaçãoalvo ${ }^{43}$. O estudo de corte transversal impede interpretar as associações encontradas como resultantes de relação de causa e efeito. $\mathrm{O}$ achado de melhor qualidade da dieta entre as pessoas com maior número de doenças crônicas é provavelmente resultante de causalidade reversa, ou seja, o surgimento das doenças levou à mudança do padrão alimentar melhorando sua qualidade.

A utilização de informação referida de doença crônica também pode ser considerada como uma limitação do estudo, porém pesquisas de comparação de informação referida e obtida em prontuários médicos mostram que o grau de acurácia varia conforme a doença pesquisada, a presença de comorbidades e as características sociodemográficas do respondente 45 . Em relação às medidas de peso e altura coletadas por mensuração direta e por relato em inquéritos nacionais, Conde et al. 47 apontaram elevadas concordância e correlação entre o IMC medido e o aferido. $\mathrm{O}$ inquérito utilizado como fonte dos dados do presente estudo abrange uma temática ampla, não sendo uma pesquisa específica de nutrição, o que reduz o detalhamento do item de consumo alimentar; contudo, por sua vez, amplia as dimensões de saúde que podem ser avaliadas em sua relação com a dieta. O IQD-R é fundamentado no instrumento americano HEI-2005, que contempla as recentes evidências científicas sobre os benefícios de certos alimentos à saúde, como os grãos integrais, os vegetais verdes-escuros e alaranjados, e óleos, incluindo os vegetais não hidrogenados e os provenientes de peixes e oleaginosas. O consumo de frutas pode ser verificado nas formas de suco natural e integral (constituição natural do alimento), e as recomendações foram definidas em $1.000 \mathrm{kcal}$, permitindo conceituar a dieta independentemente da necessidade individual de energia.

A presente pesquisa traz informações de âmbito populacional sobre a qualidade global da dieta dos idosos, identificando os componentes com piores pontuações. Os achados apontam os subgrupos mais vulneráveis ao pior padrão alimentar e a concomitância da má qualidade da dieta com outros comportamentos não saudáveis. Sinaliza, assim, para a necessidade de que as estratégias direcionadas à promoção da saúde dos idosos sejam feitas de maneira integral e não focadas em um único fator. 


\section{Resumen}

El objetivo es evaluar la calidad de la dieta en ancianos, a partir de las variables sociodemográficas, comportamientos relacionados con la salud y morbilidad. Se trata de un estudio transversal con una muestra de 1.509 ancianos, obtenidos de la encuesta de salud de Campinas, São Paulo, Brasil, en 2008-2009. La calidad de los alimentos se evaluó mediante el Índice de Calidad de la Dieta Revisado (IQD-R). Se estimaron las medias del $I Q D-R$, de acuerdo con las variables independientes $y$ un modelo de regresión lineal múltiple. Los grupos con una puntuación mayor de calidad de dieta fueron los mayores de 80 años, los evangélicos, los que hacen actividad física como hobby, y los diabéticos. Los puntajes más bajos fueron observados entre los que viven con tres o más personas, los fumadores y aquellos que informaron preferir refrescos y bebidas alcohólicas. Los resultados destacan la necesidad de mejorar la calidad de la dieta de ancianos en general y, más específicamente, para los grupos que tienen otros comportamientos no saludables, indicando la importancia de intervenciones integradas y no centralizadas.

Consumo de Alimentos; Dieta; Nutrición del Idoso; Salud del Anciano

\section{Referências}

1. Iser BPM, Yokota RTC, Sá NNB, Moura L, Malta DC. Prevalência de fatores de risco e proteção para doenças crônicas nas capitais do Brasil - principais resultados do Vigitel 2010. Ciênc Saúde Coletiva 2012; 17:2343-56.

2. Inzitari M, Doets E, Bartali B, Benetou V, Di Bari $M$, Visser $M$, et al. Nutrition in the age-related disablement process. J Nutr Health Aging 2011; 15:599-604.

3. Soenen S, Chapman IM. Body weight, anorexia, and undernutrition in older people. J Am Med Dir Assoc 2013; 14:642-8.

4. Fisberg RM, Marchioni DML, Castro MA, Verly Junior E, Araújo MC, Bezerra IN, et al. Ingestão inadequada de nutrientes na população de idosos do Brasil: Inquérito Nacional de Alimentação 2008-2009. Rev Saúde Pública 2013; 47(1 Suppl): 222S-30S.

5. Kennedy ET, Ohls J, Carlson S, Fleming K. The Healthy Eating Index: design and applications. J Am Diet Assoc 1995; 95:1103-8.

6. U.S. Department of Agriculture; U.S. Department of Health and Human Services. Dietary guidelines for Americans, 2005. Washington DC: U.S. Department of Agriculture/U.S. Department of Health and Human Services; 2005.

\section{Colaboradores}

D. Assumpção realizou a proposta do artigo, revisão da literatura, análise, interpretação dos dados e redação do texto. S. M. A. Domene colaborou na revisão do artigo. R. M. Fisberg contribuiu na revisão do artigo e na elaboração do bloco de consumo alimentar do ISA-Camp 2008. M. B. A. Barros orientou a proposta do artigo e da análise, colaborou na redação do texto e na interpretação dos dados.

\section{Agradecimentos}

Agradecimentos ao CNPq (processo no 409747/2006-8) pelo financiamento da pesquisa e pelas bolsas de produtividade de M. B. A. Barros e de R. M. Fisberg. À Secretaria Municipal de Saúde de Campinas e à Secretaria de Vigilância em Saúde do Ministério da Saúde, pelo apoio financeiro à pesquisa de campo do ISA-Camp 2008. À Capes pela bolsa de doutorado concedida a D. Assumpção.
7. Guenther PM, Reedy J, Krebs-Smith SM, Reeve $\mathrm{BB}$, Basiotis PP. Development and evaluation of the Healthy Eating Index-2005: technical report. Washington DC: Center for Nutrition Policy and Promotion, U.S. Department of Agriculture; 2007.

8. Fisberg RM, Slater B, Barros RR, Lima FD, Cesar CLG, Carandina L, et al. Índice de Qualidade da Dieta: avaliação da adaptação e aplicabilidade. Rev Nutr 2004; 17:301-8.

9. Coordenação-Geral da Política de Alimentação e Nutrição, Departamento de Atenção Básica, Secretaria de Atenção à Saúde, Ministério da Saúde. Guia alimentar para a população brasileira: promovendo a alimentação saudável. Brasília: Ministério da Saúde; 2006.

10. Previdelli AN, Andrade SC, Pires MM, Ferreira SRG, Fisberg RM, Marchioni DM. Índice de Qualidade da Dieta Revisado para população brasileira. Rev Saúde Pública 2011; 45:794-8.

11. Domene SMA. Avaliação do consumo alimentar. In: Taddei JA, Lang RMF, Silva GL, Toloni MHA, organizadores. Nutrição em saúde pública. Rio de Janeiro: Rubio; 2011. p. 41-54.

12. Fisberg RM, Villar BS. Manual de receitas e medidas caseiras para cálculo de inquéritos alimentares. São Paulo: Editora Signus; 2002. 
13. Pinheiro ABV, Lacerda EMA, Benzecry EH, Gomes MCS, Costa VM. Tabela para avaliação de consumo alimentar em medidas caseiras. 5a Ed. São Paulo: Editora Atheneu; 2004.

14. Craig CL, Marshall AL, Sjostrom M, Bauman AE, Booth ML, Ainsworth BE, et al. International physical activity questionnaire: 12-country reliability and validity. Med Sci Sports Exerc 2003; 35:1381-95.

15. World Health Organization. Global recommendations on physical activity for health. Geneva: World Health Organization; 2010.

16. Scazufca M, Menezes PR, Vallada H, Araya R. Validity of the self-reporting questionnaire-20 in epidemiological studies with older adults. Soc Psychiatry Psychiatr Epidemiol 2009; 44:247-54.

17. Cervi A, Franceschini SCC, Priore SE. Análise crítica do uso do índice de massa corporal para idosos. Rev Nutr 2005; 18:765-75.

18. Willett WC, Howe GR, Kushi LH. Adjustment for total energy intake in epidemiologic studies. Am J Clin Nutr 1997; 65(4 Suppl):1220S-8S.

19. Ervin RB. Healthy Eating Index-2005 total and component scores for adults aged 20 and over: $\mathrm{Na}$ tional Health and Nutrition Examination Survey, 2003-2004. Natl Health Stat Report 2011; 44:1-9.

20. Andrade SC. Mudanças na qualidade da dieta e seus fatores associados em residentes do Município de São Paulo em 2003-2008: estudo de base populacional [Tese de Doutorado]. São Paulo: Universidade de São Paulo; 2013.

21. Bowman SA, Lino M, Gerrior SA, Basiotis PP. The Healthy Eating Index: 1994-96. Washington DC: Center for Nutrition Policy and Promotion, U.S. Department of Agriculture; 1998.

22. Juan WY, Guenther PM, Kott PS. Diet quality of older Americans in 1994-96 and 2001-02 as measured by the Healthy Eating Index-2005. Washington DC: Center for Nutrition Policy and Promotion, U.S. Department of Agriculture; 2008. (Nutrition Insight, 41).

23. Hiza HAB, Casavale KO, Guenther PM, Davis CA. Diet quality of Americans differs by age, sex, race/ ethnicity, income, and education level. J Acad Nutr Diet 2012; 113:297-306.

24. Departamento de Análise de Situação de Saúde, Secretaria de Vigilância em Saúde, Ministério da Saúde. Plano de ações estratégicas para o enfrentamento das doenças crônicas não transmissíveis (DCNT) no Brasil 2011-2022. Brasília: Ministério da Saúde; 2011.

25. Wang Y, Chen X. How much of racial/ethnic disparities in dietary intakes, exercise, and weight status can be explained by nutrition- and healthrelated psychosocial factors and socioeconomic status among US adults? J Am Diet Assoc 2011; 111:1904-11.

26. Chen X, Cheskin LJ, Shi L, Wang Y. Americans with diet-related chronic diseases report higher diet quality than those without these diseases. J Nutr 2011; 141:1543-51.
27. Lima-Costa MF. A saúde dos adultos na Região Metropolitana de Belo Horizonte: um estudo epidemiológico de base populacional. Belo Horizonte: Núcleo de Estudos em Saúde Pública e Envelhecimento, Fundação Oswaldo Cruz/Universidade Federal de Minas Gerais; 2004.

28. Instituto Brasileiro de Geografia e Estatística. Censo Demográfico 2010: características gerais da população, religião e pessoas com deficiência. Rio de Janeiro: Instituto Brasileiro de Geografia e Estatística; 2010.

29. Sarri KO, Higgins S, Kafatos AG. Are religions "healthy"? A review on religious recommendations on diet and lifestyle. J Hum Ecol 2006; 14:7-20.

30. Instituto Brasileiro de Geografia e Estatística. Censo Demográfico 2010: famílias e domicílios. Rio de Janeiro: Instituto Brasileiro de Geografia e Estatística; 2010.

31. Borim FSA, Barros MBA, Neri AL. Autoavaliação da saúde em idosos: pesquisa de base populacional no Município de Campinas, São Paulo, Brasil. Cad Saúde Pública 2012; 28:769-80.

32. Lebrão ML, Laurenti R. Saúde, bem-estar e envelhecimento: o estudo SABE no Município de São Paulo. Rev Bras Epidemiol 2005; 8:127-41.

33. Xu B, Houston DK, Locher JL, Ellison KJ, Gropper S, Buys DR, et al. Higher Healthy Eating Index-2005 scores are associated with better physical performance. J Gerontol A Biol Sci Med Sci 2012; 67:93-9.

34. World Health Organization. Global status report on noncommunicable diseases 2010. Geneva: World Health Organization; 2011.

35. Observatório da Política Nacional de Controle do Tabaco. A política nacional. http://www2.inca.gov. $\mathrm{br} / \mathrm{wps} / \mathrm{wcm} /$ connect/observatorio_controle_ta baco/site/status_politica/a_politica_nacional (acessado em 15/Dez/2012).

36. Departamento de Desenvolvimento Humano, Banco Mundial. Documento de discussão - saúde, nutrição e população (HNP) controle do tabagismo no Brasil. Brasília: Banco Mundial; 2007.

37. McClernon FJ, Westman EC, Rose JE, Lutz AM. The effects of foods, beverages, and other factors on cigarette palatability. Nicotine Tob Res 2007; 9:505-10.

38. Haibach JP, Homish GG, Giovino GA. A longitudinal evaluation of fruit and vegetable consumption and cigarette smoking. Nicotine Tob Res 2013; 15:355-63.

39. Monteiro CA, Castro IRR. Por que é necessário regulamentar a publicidade de alimentos. Ciênc Cult (São Paulo) 2009; 61:56-9.

40. Instituto Brasileiro de Geografia e Estatística. Pesquisa de Orçamentos Familiares 2008-2009: análise do consumo alimentar pessoal no Brasil. Rio de Janeiro: Instituto Brasileiro de Geografia e Estatística; 2011. 
41. Bueno MB, Marchioni DML, César CLG, Fisberg RM. Added sugars: consumption and associated factors among adults and the elderly. São Paulo, Brazil. Rev Bras Epidemiol 2012; 15:256-64.

42. Thomson JL, Tussing-Humphreys LM, Onufrak SJ, Connell CL, Zoellner JM, Bogle ML, et al. Simulated reductions in consumption of sugar-sweetened beverages improves diet quality in Lower Mississippi Delta adults. Food Nutr Res 2011; 55:7304.

43. Breslow RA, Guenther PM, Juan W, Graubard BI. Alcoholic beverage consumption, nutrient intakes, and diet quality in the US adult population, 19992006. J Am Diet Assoc 2010; 110:551-62.

44. Herbeth B, Samara A, Stathopoulou M, Siest G, Visvikis-Siest S. Alcohol consumption, beverage preference, and diet in middle-aged men from the STANISLAS study. J Nutr Metab 2012; 2012:987243.
45. Barros MBA, Francisco PMSB, Zanchetta LM, César CLG. Tendências das desigualdades sociais e demográficas na prevalência de doenças crônicas no Brasil, PNAD: 2003-2008. Ciênc Saúde Coletiva 2011; 16:3755-68.

46. Lima-Costa MF, Matos DL, Camargos VP, Macinko J. Tendências em dez anos das condições de saúde de idosos brasileiros: evidências da Pesquisa Nacional por Amostra de Domicílios (1998, 2003, 2008). Ciênc Saúde Coletiva 2011; 16:3689-96.

47. Conde WL, Oliveira DR, Borges CA, Baraldi LG. Consistência entre medidas antropométricas em inquéritos nacionais. Rev Saúde Pública 2013; 47:69-76.

Recebido em 22/Jan/2013

Versão final reapresentada em 23/Dez/2013

Aprovado em 05/Fev/2014 